\title{
Design and Analysis of Two Six-port Correlators
}

\author{
Engr. Muhammad Irfan Khan ${ }^{1}$, Dr. Syed Waqar Shah ${ }^{2}$ \\ ${ }^{\prime}$ (Department of Electrical Engineering, University of Engineering \& Technology, Peshawar, Pakistan) \\ ${ }^{2}$ (Department of Electrical Engineering, University of Engineering \& Technology, Peshawar, Pakistan)
}

\begin{abstract}
This paper presents design and analysis of two different Ultra Wide band (UWB) six-port correlators for transmitter and receiver applications. The two designs analyzed and compared in terms of transmission parameters, relative bandwidth and occupied area. On one side, design of microwave components for multiGiga Hertz bandwidth is a non-trivial challenge. Meeting such challenging performance specifications requires a high degree of Electronic Design Automation (EDA)-tool skills and theoretical understanding of the limits and possibilities offered by the used circuit implementation. On the other side, an appropriate design methodology must be established and systematically used along the entire project development. Two different designs of a sixport correlator for more than $3 \mathrm{GHz}$ frequency bandwidth are presented. The systematic design approach applied in the case of the six-port correlator can be extrapolated for any microwave circuit using passive components with transmission lines.
\end{abstract}

Keywords: Correlator, Transmission lines, Ultra wide Band (UWB)

\section{Introduction}

A lot of research activity in the field of Ultra-wideband (UWB) circuit and communication system design was and is performed within the academia and industry. Among the possible ways to implement UWB transceivers for high data rates, the six-port transceiver using the six-port correlator has shown very good performances with low consumption power, relative simplicity at circuit level and low manufacturing cost. However, these advantages can be achieved only by using accurate and appropriate design methodology using complex design tools.

Six-port technology surfaced as an elegant means for measuring microwave quantities in 1960's and 1970's. Later on it was used in RADAR applications. It was very recently that six-port technology was used for constructing communication receivers [1]. The use of six-port as a communication receiver was initiated for the first time in 1994 by Ji. Li, R. G. Bosisio and Ke Wu [2]. Being a wideband solution, six-port still suffers with the problem of limited bandwidth. These days UWB greater than $40 \%$ of relative bandwidth is needed to achieve high speed over short ranges for a wireless link [3].

\section{Basic building blocks and operation}

The Six-port transceiver is a passive RF circuit. The basic building blocks are composed of three $90^{\circ}$ hybrid couplers and one $3 \mathrm{~dB}$ Wilkinson power divider as shown in Fig 1.

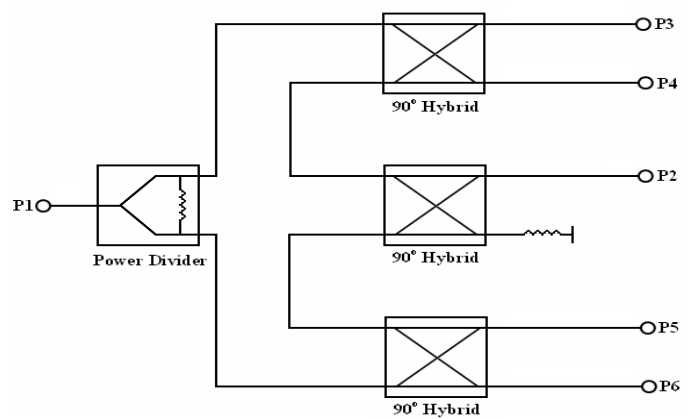

Figure 1 Block diagram of a typical six-port circuit

It has seven ports but only six are used. When used as a transmitter, port 1 is fed with local oscillator (LO) signal, port 2-3 and port 5-6, usually terminated with switching devices, are fed with baseband data while the output signal. i.e; RF signal is taken from port 4. In receiver mode, portl is fed with LO signal, port 4 with RF signal while port 2-3 and port 5-6 are used for demodulating the received signal using a switch which in most cases is an active component. 


\section{III. ultra wideband (UWB)}

Ultra wide band is extremely wide spread spectrum where RF energy is spread over gigahertz of spectrum. It is wider than any narrowband system by orders of magnitude. It can be loosely defined as any wireless transmission scheme that occupies a bandwidth of more than $25 \%$ of a center frequency, or more than $1.5 \mathrm{GHz}$. UWB uses coded pulses of extremely short duration. UWB signals can be designed to appear as random noise spread across a wide spectrum. Fig 2 shows the difference between narrowband and UWB communication. UWB occupies more than $500 \mathrm{MHz}$ of spectrum.

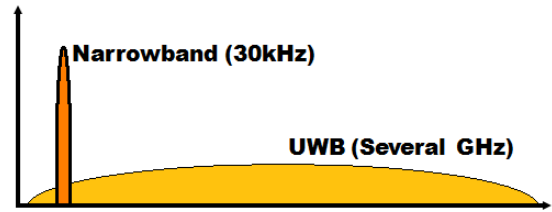

Figure 2 Comparison of UWB and narrowband Spectrum

\section{Designing The Six Port Correlator}

Two designs were made for a six-port transceiver, one; a conventional design and other with external matching networks. First, a conventional design was made and then another design with external matching networks was made. The results deviated from expectations when power divider and couplers are connected together. Thus, optimization was needed at microstrip schematic level. Line parameters had to be adjusted at the same time maintaining perfect geometry of the six-port circuit at layout level. Fig 3 shows a flow chart indicating different stages during design process of both the six-port transceivers.

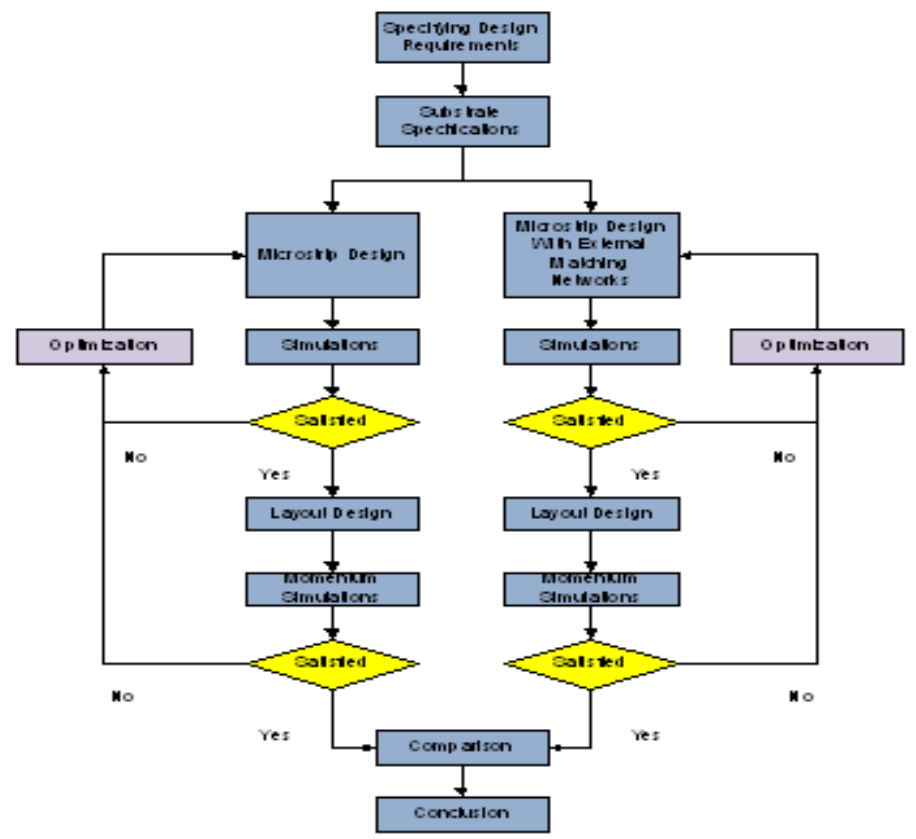

Figure 3 Design methodology of six-port correlators

\section{Conventional six port correlator}

The design was made quite easily by connecting three identical couplers to each other and to a power divider in such a fashion that it would occupy minimum possible area on the PCB. The microstrip design of the six-port coupler is shown in Fig 4. 


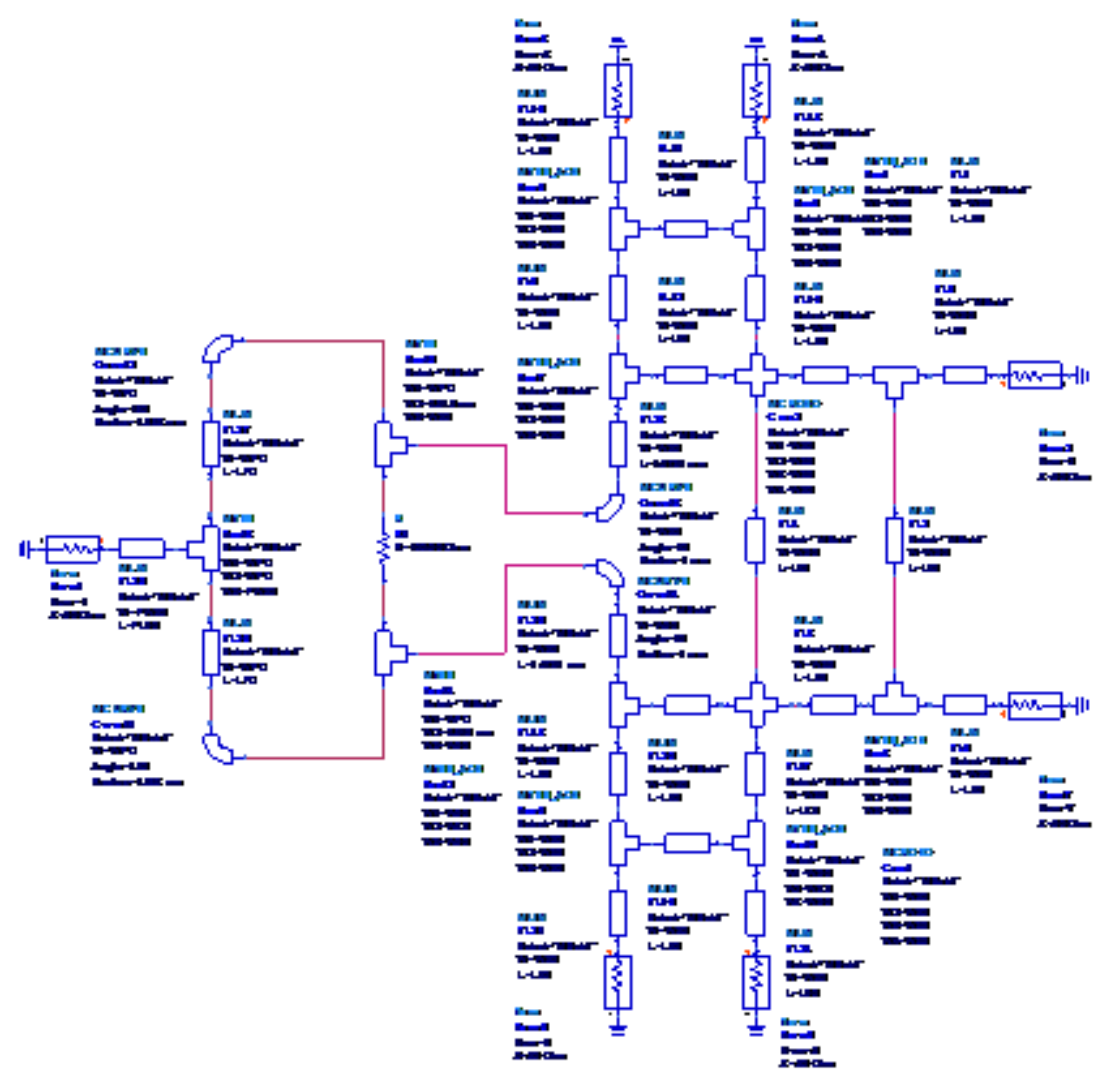

Figure 4 Schematic of conventional six-port correlator

Curves were used to connect the couplers to the power-divider. Bends or Corns were avoided as the curves have the property of generating smooth plots. Port 1 can act as an LO input while port 2 as an RF output for a transmitter. In case of a receiver, port 2 will act as an RF input while Port 1 remains as an LO input.

The design was simulated from 5.5 to $9.5 \mathrm{GHz}$. Fig 5 and 6 show the results for amplitude response, amplitude imbalance, phase response and phase imbalance at all the output ports.

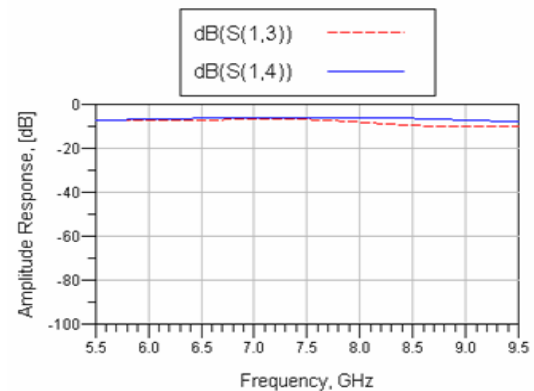

(a)

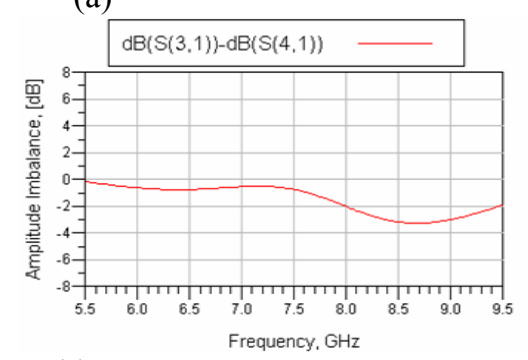

(c)

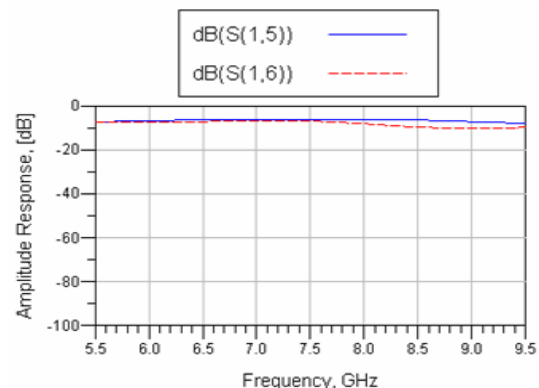

(b)

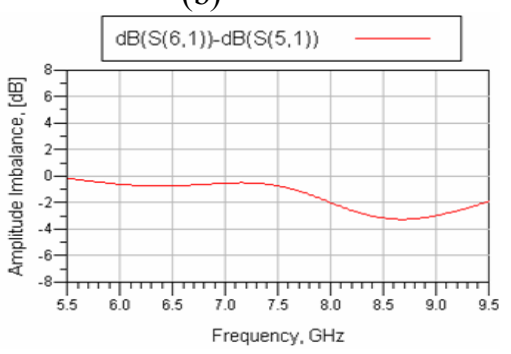

(d)

Figure 5 (a) (b) Amplitude Response (c) (d) Amplitude imbalance 


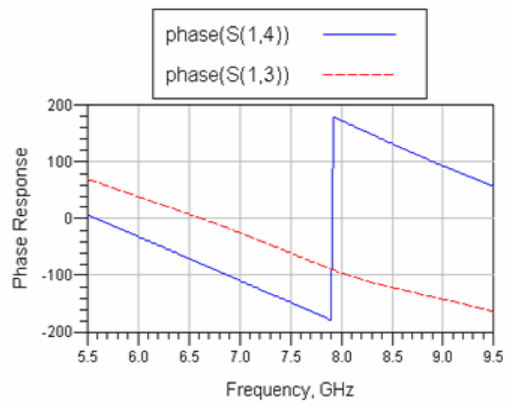

(a)

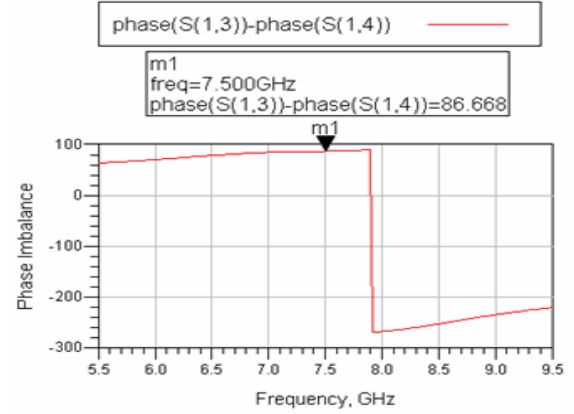

(c)

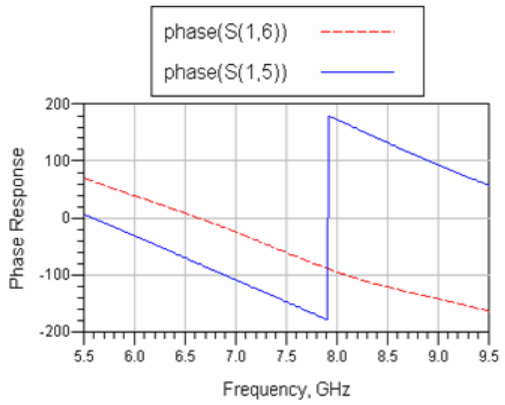

(b)

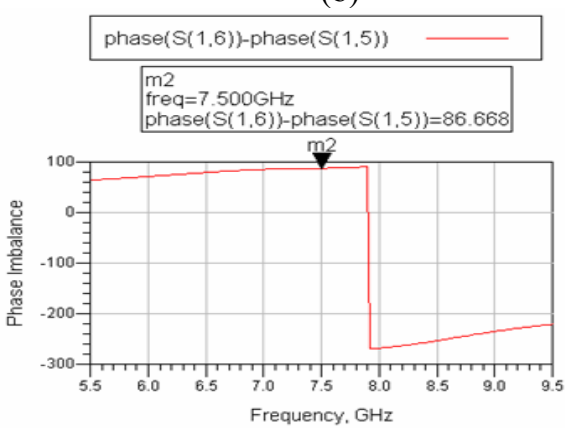

(d)

Figure 6 (a) (b) Phase response (c) (d) Phase imbalance

\section{Six-Port Correlator With External Matching Networks}

Three identical quadrature hybrids with external matching networks connected with a Wilkinson power divider for design a six-port correlator with external matching networks. The impedance of both Zline and Zstub being 1.33Zo. The addition of Zline and Zstub shown in Fig 7 to the six-port correlator gives one a lot of flexibility in the design process.

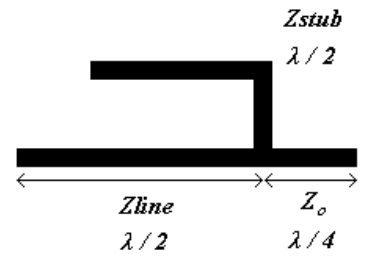

Figure 7 Design of external matching network

Fig 8 shows a microstrip schematic design of a six-port correlator. Note that couplers are connected here in different geometry as compared to the six-port design shown in Fig 5 


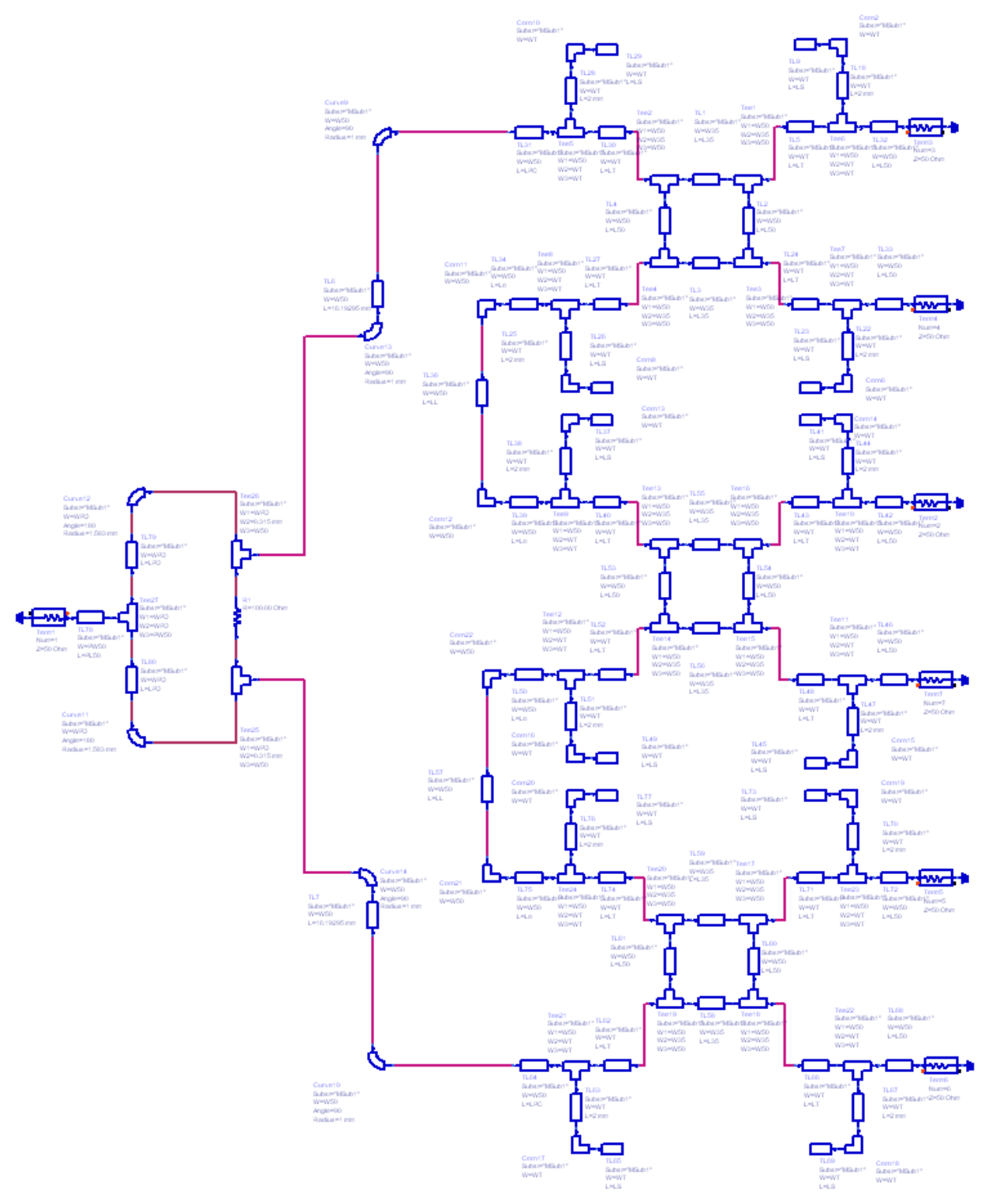

Figure 8 Schematic of six-port correlator with external matching networks

All the outputs are now on same side in order to keep the size of PCB as small as possible. But for the purpose of PCB manufacturing, the output terminations have to be given some bend in order to make some space for the connectors which is quite a hectic job and results will need to be tuned again. In order to cover lesser space on PCB, another design with stubs oriented towards inside was also tried but could not be implemented efficiently due to lack of time.

Fig 9 and 10 show the simulation results for amplitude response, amplitude imbalance, and phase response and phase imbalance at all the output ports. 


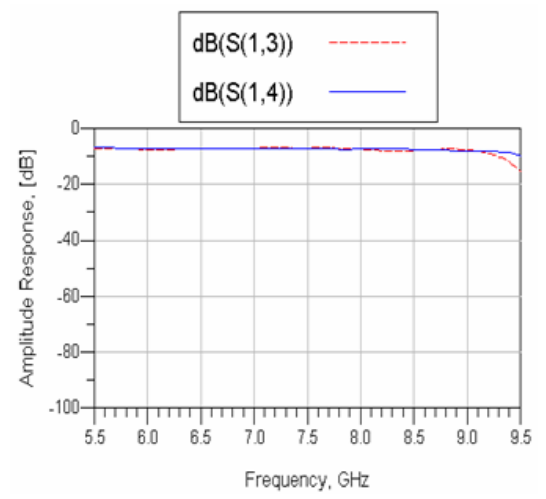

(a)

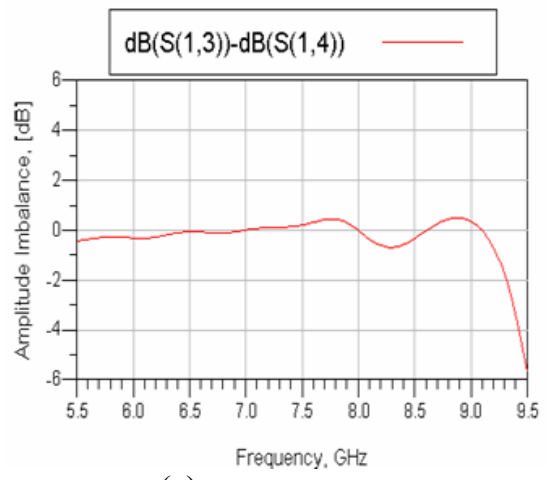

(c)

Figure 9 (a) (b) Amplitude response

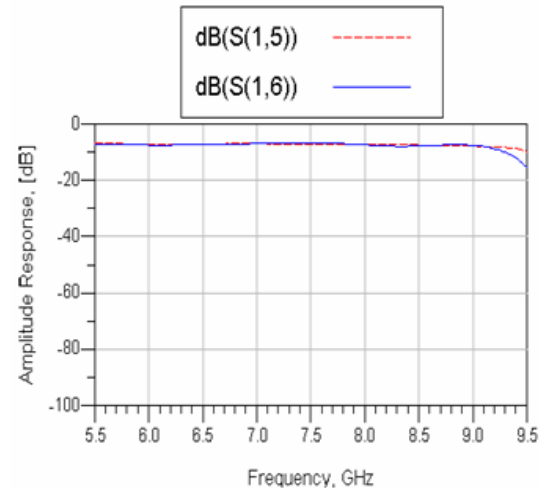

(b)

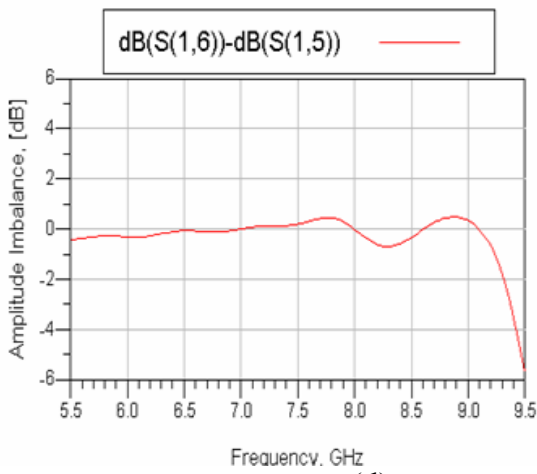

(d)

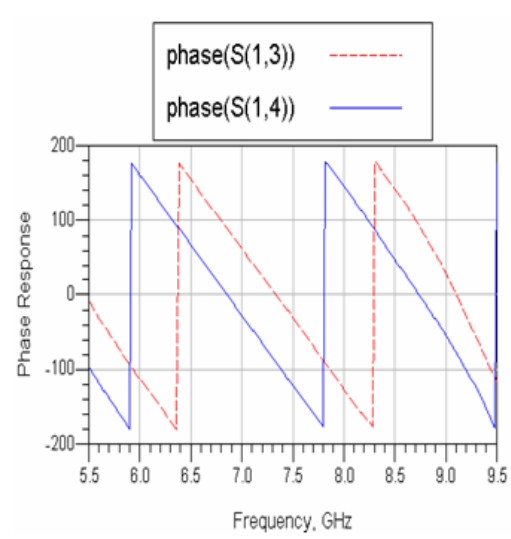

(a)

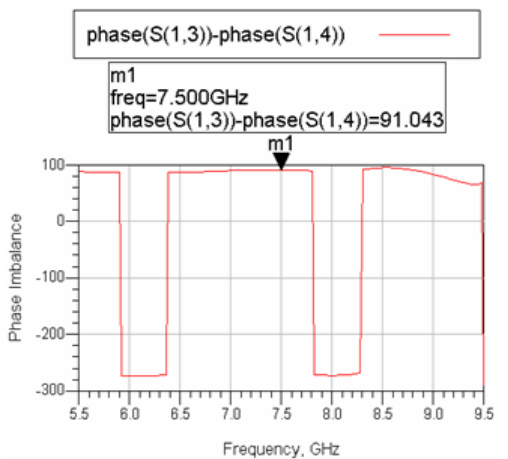

(c)

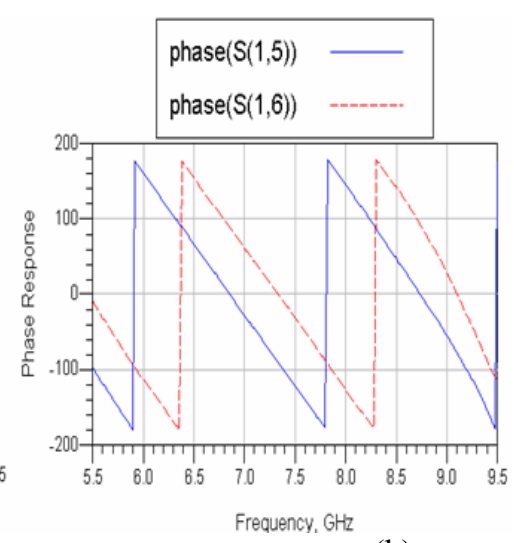

(b)

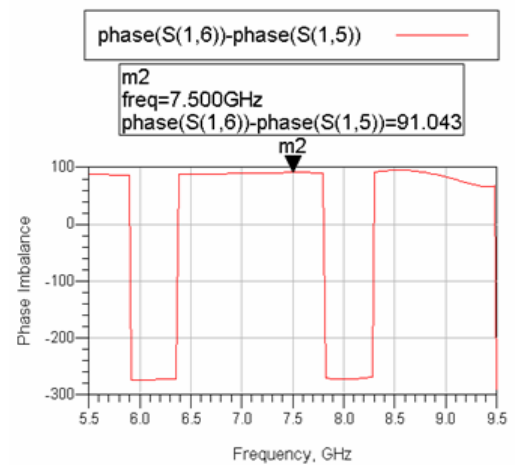

(d)

Figure 10 (a)(b) Phase response (c)(d) Phase imbalance 


\section{Conclusion}

The relative bandwidth of a quadrature hybrid is about 10 to $15 \%$ but can be increased up to $50 \%$ or even more with the addition of external matching networks. The amplitude response becomes much flatter with external matching networks. The same flatness is evident when the couplers with external matching networks are combined with Wilkinson power divider to make a complete six-port circuit. A flat amplitude response was noticed. Also, amplitude imbalance remains between $1 \mathrm{~dB}$ over major portion of the bandwidth. The phase imbalance in both designs remains almost around 90o but the addition of external matching networks keeps the response much flatter at higher frequencies. Both designs had its pros and cons. While the correlator with external matching networks improved relative bandwidth, the conventional six-port correlator would occupy lesser space and reduce PCB size by $57 \%$. Also, it was much simpler to design a conventional six-port correlator; whereas, the design with external matching networks took a lot of time in optimization.

The addition of external matching networks with Zline and Zstub equal to $1.33 \mathrm{Zo}$, gives a designer the flexibility and ease of improving the relative bandwidth by mere tuning of the stubs.

The goal was to design these two correlators of different structural dimensions by following an understood design methodology. It was not an easy task to design two different six-pot correlators and compare them, particularly when power divider had to be connected to the quadrature hybrids. But, by following a clear and step-wise design methodology, the task was made much simpler and disciplined. This methodology divided the entire design into smaller designs which were independently created at different levels of abstraction (i.e; ideal, microstrip and layout) and then connected with other components. An iterative process of optimization, outlined between microstrip schematic level and layout level, was already anticipated. Thus, following a clear and distinct design methodology is an essential part of any RF system design project.

\section{References}

[1]. The Six-Port as a Communications Receiver, Tim Hentschel, Microwave Theory and Techniques, IEEE Transactions on Microwave Theory and Techniques, Volume: 53, Issue: 3, Part: 2

[2]. J. Li, R.G. Bosisio and K. Wu "Computer and Measurement Simulation of a New Digital Receiver Operating Directly at Millimeter-Wave Frequencies", IEEE Trans. Microw. Theory Tech., Vol 43, No 12, pp.2766-2772, 1995

[3]. Ultra-Wideband Six-port Transmitter and Receiver Pair 3.1-4.8 GHz Pär Håkansson and Shaofang Gong Linköping University, Department of Science and Technology SE-601 74 Norrköping, Sweden

[4]. David. M. Pozar, Microwave Engineering, John Wiley \& Sons, Inc, 2005, 3rd edition, p.318-333

[5]. E. Wilkinson, “An N-way hybrid power divider,” IRE Trans. Microw. Theory Tech., vol. MTT-8, no. 1, pp. 116-118, Jan. 1960.

[6]. S. B. Cohn, "A class of broadband three port TEM-mode hybrids," IEEE Trans. Microw. Theory Tech., vol. MTT-16, no. 2, pp. $110-116$, Feb, 1968

[7]. Parad and Moynihan, "Split-tee power divider," IRE Trans. Microw. Theory Tech., pp. 91-95, Jan. 1965.

[8]. Youngkwang Lim; Hai-young Lee; "Novel miniaturized branch-line coupler using bond-wire slow-wave structure," Microwave Conference, 2008. APMC 2008. Asia-Pacific, vol., no., pp.1-4, 16-20 Dec. 2008]

[9]. M. Muraguchi, T. Yukitake, Y. Naito, "Optimum design of $3 \mathrm{~dB}$ branch-line couplers using microstrip lines", IEEE Trans. Microwave Theory and Tech., vol. 31, pp.674-678, 1983.

[10]. M-PSK and M-QAM Modulation/Demodulation of UWB Signal Using Six-Port Correlator, Negar Sani, ITN Department , Linköping University

[11]. Hoer, C. A., "The Six-Port Coupler: A New Approach to Measuring Voltage, Current, Power, Impedance and Phase," IEEE Trans. IM, Vol. IM-21, 1972, pp. 466-470.

[12]. Engen, G. F., "The Six-Port Reflectometer: An Alternative Network Analyzer," IEEE Trans. Microwave Theory and Tech., Vol. MTT-25, pp. 1075-1079, 1977.

[13]. Oishi, T., and W. W. Kahn, "Stokes Vector Representation of the Six-Port Network Analyzer: Calibration and Measurement," MTT International Microwave Symposium, June 1985, St.Louis, MO, pp. 503-506.

[14]. Fadhel M. Ghannouchi, Abbas Mohammadi, The Six-Port Technique with Microwave and Wireless Applications, 2009 ARTECH HOUSE 